\title{
Editorial: Time in Electroacoustic Music
}

This issue addresses the multifaceted topic of time in electroacoustic music. The subject is of course a vast one, as all music is by nature temporal. In Western classical traditions, the various manifestations of time ended up being notated through a plethora of conventions: tempo markings, note durations, bar lines, timbre (expressed through a combination of instrumentation, dynamics, articulation and ornamentation but usually with the finer details supplied by the performer). As a result, the few discussions of temporal properties of music beyond the pitch-centred harmonic/melodic texts were usually subsumed into discussions of metre, rhythm and, sometimes, large-scale formal design.

Interest in the topic of time itself was rekindled in the early twentieth century - in contexts from physics to philosophy - and increasingly, experimentation with new forms of organising temporal flow and duration in music became more commonplace, with the prominence of works by composers ranging from Stravinsky, Webern and Varèse, to Cage, Carter and Xenakis (to name a few). The growing participation of electroacoustic musicians in this emerging group is no surprise, as the freedom from notation instantly distanced them from notation-based frameworks for creation and discussion. In addition, direct references to 'clock time' became more overt, whether through measuring sounds by tape length or providing instructions to a computer in terms of milliseconds and frequencies, thus linking in to the measuring tools of scientific data. Moreover, the ability to delve into the microscopic allowed the curious to dissect sounds-sometimes in the context of timbre research and sound synthesis - and construct new material which might or might not share any resemblance to more familiar sources. Issues of perception began to be studied with reference to emerging research in acoustics and information theory, as in the case of Meyer-Eppler's work in the Cologne lab, propagated in part through Stockhausen.

Thus, for many composers and researchers, the manifestation and behaviour of various temporal aspects, and even speculations about the nature of time itself, became rewarding topics to explore through reflection, discussion and compositional manipulation. Some of the aspects can be (and have been) dealt with under the term 'rhythm', but the multiple meanings of that term are not always acknowledged, nor is it usefully applied to the timbral level - even though electroacoustic composers are quite aware that audible rhythms can be easily transformed into timbre, and vice versa, by global temporal expansion or contraction. Nevertheless, the discussion continues to be somewhat ad hoc; the multiple facets of musical time are still rarely addressed as a coherent set of properties in musicological or analytical contexts, with notable exceptions by authors such as Kramer, Rowell, Becker and Tenney. Until the various contributions become more widely known, commented on and eventually integrated into introductory courses in music analysis and composition, we lack an accepted set of cross-stylistic terms and concepts for categorising temporal attributes.

While the term 'rhythm' may seem unwieldly in music analysis, 'time' itself is even more prone to multiple interpretations. This is doubtless due partly to the fact that we all know time is an absolutely essential ingredient of our lives, but we are not handed any simple definitions of what it is; nor can we see it. Musicians are better prepared for such difficulties than most, because we cannot see sound, either - but we know that it is real, and able to be studied. We are quite used to visual cues such as spectrograms and amplitude graphs which specify every millisecond of a given audio passage in precise order, and we know how to zoom in and out-so we learn something of how the microscopic and the macroscopic may relate to each other - if we are focusing on the linear sequential ordering of the sounds. We know that those measurements correspond precisely to our watches and computer clocks. However, many composers and analysts appreciate that, despite such precision, the clock-time visualisation of a piece does not completely or accurately reveal our perception of its temporal characteristics or flow. This has led many (including some of the authors in this issue) to a reflection on the dichotomy (as it is usually viewed) between 'objective' time as displayed by the clock, and 'subjective' or 'experiential' time, which is how that time appears to us as individuals. On the other hand, there are also quite different perspectives which argue that the main dichotomy is that of the cyclic (time's cycle) vs the linear views of time (time's arrow); or the emphasis on duration vs succession, continuous vs discrete. Issues of memory, for example, operate on several levels, and are dependent on the complex issues of cognitive processing. Scale and focus are clearly relevant as well: in the often-abstract world of electroacoustics, the listener may opt for a 'close-up' or a 'distance' view of the sonic configurations with quite different results which may or may not have been anticipated by the composer.

Depending on one's perspective and interests, therefore, several ways exist for grouping the articles in this 
issue. The grouping we have chosen for the print order roughly divides the articles into two categories: those exploring time in electroacoustic music from the perspective of reception and the others opting for exploring composers' temporal organisational practices. Of course, it goes without saying that both viewpoints are not mutually exclusive; the common link being, of course, the necessary degree of subjectivity when addressing the question of time in music.

Apart from the specific composers highlighted in the first grouping of articles, several authors clarify how their particular approaches incorporate or distinguish themselves from other related discussions. Thus we have reference to - and commentary on - concepts and terms from various disciplines (musicology, cognitive science, philosophy, etc.) and authors (Varèse, Cage, Xenakis, Schaeffer, Huron, Bergson, Scruton, Strawson, Roads, Bregman, etc.) to help the reader appreciate the range of thinking emerging from decades of reflection and experimentation. Other authors have contributed to this purpose more obliquely, but equally persuasively, by revealing their own personal approaches to the subject - as composers, listeners and musicologists. Thus, we find stimulating reflections on key terminology and concepts including rhythm, memory, anticipation, timescales and polychrony.

All the articles, in one way or another, aim to provide frameworks for deeper discussions of time in music through very diverse methodologies: from the theoretical to the empirical, from musicological analyses to psychological experiments, and from an examination of and speculation about classic and novel compositional approaches, listening strategies and terminologies within the field of electroacoustic music in its broadest sense. This diversity is, of course, welcome, and vividly demonstrates that the 'time of music' is still a largely uncharted territory: we hope that this issue will help in guiding the interested explorer.

Late twentieth-century research on musical perception has a strong reliance on Western classical music language and more often than not on tonal music. In their article, Vincenzo Santarcangelo and Riccardo Wanke set out to examine how this may work in the electroacoustic world. Using extensive contemporary music examples, they demonstrate how the early stages of perception seem to unearth 'primal sound configurations' which are then stored in memory.

By focusing on the compositional notes and manuscripts of spectral compositions by Gérard Grisey and Kaija Saariaho, José Besada and Cristóbal Pagán Cánovas propose that attention to the composer's cognitive activity can help to establish a common language for the representation of time in electroacoustic and contemporary music. They show how composers, through the integration of various visual notations (from traditional scores to 3D spectrograms), can establish complex creative strategies for escaping traditional temporal conceptions.

In a study of seminal works by Stockhausen and Berio, Danilo Rossetti, Micael Antunes and Jônatas Mazolli examine how the sense of direction of time can be constructed in electroacoustic music. They present a novel and coherent analytical apparatus which leads to compelling results on the direction of time in electroacoustic composition.

When discussing time in electroacoustic music, the topic of microsounds and granular synthesis often comes up in the discussion. İpek Görgün delves into Horacio Vaggione's music and written works to explore how the composer's personal concepts of objects, singularities and microsounds interact within his compositional practice.

Equipped with Paul Ricœur's concept of mimesis, Eric Maestri proposes a glimpse into how composers address the issue of temporal organisation and its conveyance to the listener through the interviews of five composers. The diversity of answers he receives show the range of attitudes when it comes to the question of conveying meaning in contemporary music.

Many of the problems addressing time in music are linked, in one way or another, to that of musical representation. The article by Cat Hope explores the current state of the use of animated notation for contemporary music, demonstrating, through diverse examples, how digital technology can facilitate and stimulate fruitful and cross-stylistic collaborations.

In a more 'hands-on' article, Felipe Otondo and Victor Poblete demonstrate the use of a fascinating time-lapse algorithm for soundscape compositions. The described approach is not only a technical investigation of modern granular synthesis techniques, but also has the potential to form the basis for educational and creative tools.

Michael Gatt's article describes a framework based on the impact of listening to acousmatic music on temporal flow. It is notably based on the exploration of the role of memory in electroacoustic music, questions the notions of style in this context and develops an engaging approach to the listening experience based on 'expectation' in relation to the connections and spectromorphological evolutions of gestures and textures.

James Andean explores the question of rhythm in acousmatic music through an ecological lens. The underlying question here is that of the conveyance of meaning in acousmatic music - the rhythmic aspects at play are viewed through music cognition and perception as a crucial element in the understanding and appreciation of acousmatic work.

Studying the idea of time-scales from the composer's perspective, Aki Pasoulas proposes a useful model to 
illustrate his explanation of the mutual influence of absolute and psychological times and their impacts on electroacoustic and sound art.

Jason Noble, Tanor Bonin and Stephen McAdams present an article well grounded in the principles of auditory perception, and, through an in-depth psychological experiment of listeners' reactions to various types of music, show how some electroacoustic music has exceptional potential to be gauged as inherently timeless.

Kevin Dahan elaborates the concepts of sonic unit, temporal directionality and distancing, and how in their organisational structuring at different temporal levels, they can create the sense of time moving at different rates: a principle identified by the author as polychrony.

Rosemary Mountain uses a variety of whimsical analogies to illustrate the many variables in our temporal focus, and proposes that our auditory system can be usefully viewed as containing adjustable arrays of receptors for identifying and tracking disparate elements in the music.

The variety of topics contained here is clearly not exhaustive, but we are confident that it provides a good sampling of the many ways in which time intersects directly with music creation and reception.
Although the various perspectives are quite distinct, we are pleased to note that there are few discrepancies at the level of the basic assumptions and claims. Where discrepancies are discovered - either between articles or between an article and the reader's own thoughts on the matter - then we trust that the clarity of the arguments will help isolate the problematic issues and inspire further reflection. Moreover, the collective grouping of the various sources cited will provide any curious researcher with ample information to spur them on to further investigation. We trust that the articles here will prompt more participants to think deeply about time, and help them to find innovative ways to explore time within creative and/or analytical projects.

We would also like to thank all the contributors, the reviewers and the editor of the journal, Leigh Landy, for their enthusiasm and dedication to this continuing exploration.

Rosemary Mountain (rosemary.mountain@concordia.ca)

Kevin Dahan (kdahan@dmu.ac.uk) 